\title{
Genomic prediction for Nordic Red Cattle using one-step and selection index blending
}

\author{
G. Su, ${ }^{\star 1}$ P. Madsen, ${ }^{\star}$ U. S. Nielsen,† E. A. Mäntysaari,‡ G. P. Aamand,§ O. F. Christensen, ${ }^{\star}$ and M. S. Lund \\ *Department of Molecular Biology and Genetics, Faculty of Science and Technology, Aarhus University, DK-8830 Tjele, Denmark \\ †Danish Agricultural Advisory Service, DK-8200 Aarhus N, Denmark \\ ¥Biotechnology and Food Research, MTT Agrifood Research, 31600 Jokioinen, Finland \\ §Nordic Cattle Genetic Evaluation, DK-8200 Aarhus N, Denmark
}

\section{ABSTRACT}

This study investigated the accuracy of direct genomic breeding values (DGV) using a genomic BLUP model, genomic enhanced breeding values (GEBV) using a one-step blending approach, and GEBV using a selection index blending approach for 15 traits of Nordic Red Cattle. The data comprised 6,631 bulls of which 4,408 bulls were genotyped using Illumina Bovine SNP50 BeadChip (Illumina, San Diego, CA). To validate reliability of genomic predictions, about $20 \%$ of the youngest genotyped bulls were taken as test data set. Deregressed proofs (DRP) were used as response variables for genomic predictions. Reliabilities of genomic predictions in the validation analyses were measured as squared correlations between DRP and genomic predictions corrected for reliability of DRP, based on the bulls in the test data sets. A set of weighting (scaling) factors was used to construct the combined relationship matrix among genotyped and nongenotyped bulls for one-step blending, and to scale DGV and its expected reliability in the selection index blending. Weighting (scaling) factors had a small influence on reliabilities of GEBV, but a large influence on the variation of GEBV. Based on the validation analyses, averaged over the 15 traits, the reliability of DGV for bulls without daughter records was 11.0 percentage points higher than the reliability of conventional pedigree index. Further gain of 0.9 percentage points was achieved by combining information from conventional pedigree index using the selection index blending, and gain of 1.3 percentage points was achieved by combining information of genotyped and nongenotyped bulls simultaneously applying the onestep blending. These results indicate that genomic selection can greatly improve the accuracy of preselection for young bulls in Nordic Red population, and the one-

Received August 5, 2011.

Accepted October 13, 2011.

${ }^{1}$ Corresponding author: guosheng.su@agrsci.dk step blending approach is a good alternative to predict GEBV in practical genetic evaluation program.

Key words: genomic prediction, model comparison, pedigree index, reliability

\section{INTRODUCTION}

Genomic selection has been widely applied in dairy cattle breeding (Hayes et al., 2009a; VanRaden et al., 2009; Harris and Johnson, 2010; Su et al., 2010; VanRaden and Sullivan, 2010). Currently, a typical genomic evaluation in dairy cattle involves several steps. First, pseudo-observations are derived from raw data. For example, traditional EBV, daughter deviations or deregressed proofs (DRP) can be used as pseudoobservations. Estimated breeding values and daughter deviations are usually obtained from a BLUP model that integrates a pedigree-based relationship matrix. Deregressed proofs (Goddard, 1985; Schaeffer, 1985) can be derived from EBV and the effective daughter contributions (Jairath et al., 1998; Schaeffer, 2001). Second, direct genomic breeding values (DGV) are predicted using a genomic prediction model from pseudo-observations of reference animals and genome-wide SNP markers. Finally, DGV are combined with traditional parent averages (PA) or pedigree indexes (PI) to obtain genomic enhanced breeding values (GEBV).

Many statistical models have been proposed to predict DGV, which differ in the assumption of distributions of SNP effects. A linear BLUP approach (Meuwissen et al., 2001; VanRaden, 2008; Goddard and Hayes, 2009; Hayes et al., 2009b) assumes that effects of all SNP are normally distributed with equal variance. BayesA and similar approaches (Meuwissen et al., 2001; Meuwissen and Goddard, 2004; Su et al., 2010) assume that variances of SNP effects differ among loci. BayesB and other variable selection approaches (Meuwissen et al., 2001; Meuwissen and Goddard, 2004; Villumsen et al., 2009; Su et al., 2010) assume heterogeneous variances of SNP effects, with most SNP having zero or very small effects and a few having moderate to large effects. 
Simulation studies with the assumption that few QTL have a large effect and most QTL have a small effect have shown that the predictive ability of BayesA and BayesB was better than BLUP approaches (Meuwissen et al., 2001; Lund et al., 2009; Guo et al., 2010). However, experiences with real dairy cattle data indicate that limiting the number of SNP markers to only those with large effects has resulted in reduced accuracy (Cole et al., 2009; Su et al., 2010), and that a linear BLUP model performed well for most traits (Hayes et al., 2009a; VanRaden et al., 2009). Linear BLUP models (at either the SNP level or the individual animal level) have become popular approaches in practical genomic evaluations because they are simple and have low computational requirements.

The accuracies of genomic predictions can be improved by combining information of traditional EBV (PA or PI). VanRaden et al. (2009) proposed a blending approach using a selection index that includes DGV, traditional PA (or PI) calculated from the whole population, and PA (or PI) calculated from the data of genotyped animals only. A more sophisticated approach is to predict GEBV by integrating genomic, pedigree, and phenotype information in a single-step procedure (Legarra et al., 2009; Misztal et al., 2009; Aguilar et al., 2010; Christensen and Lund, 2010). However, this single-step approach might have a high computational demand in the cases of large data sets when applying complex models (e.g., multi-trait test-day model), and might not easily be implemented in genomic prediction using a combined reference population that includes genotyped foreign bulls, such as the Eurogenomics reference population (Lund et al., 2010).

A compromise between efficient blending and efficient implementation is to apply the methodology of the single-step procedure (Misztal et al., 2009; Aguilar et al., 2010; Christensen and Lund, 2010) but using DRP or daughter deviations of bulls as response variable, instead of raw data. In this context, the term "onestep blending" was used to distinguish from the original single-step procedure. The objective of this study was to investigate the reliability and unbiasedness of GEBV using the one-step blending approach for Nordic Red Cattle (RDC) and compare it with DGV from a linear genomic BLUP model (GBLUP) and GEBV from a selection index blending approach.

\section{MATERIALS AND METHODS}

\section{Data}

Estimated breeding values from the Nordic genetic evaluation in October 2010 were used to derive DRP (Jairath et al., 1998; Schaeffer, 2001) for 15 traits
Table 1. Number of bulls in total reference data set $\left(\mathrm{REF}_{t}\right)$, genotyped reference data set $\left(\mathrm{REF}_{\mathrm{s}}\right)$, and test data set

\begin{tabular}{llll}
\hline Trait & $\mathrm{REF}_{\mathrm{t}}$ & $\mathrm{REF}_{\mathrm{s}}$ & Test \\
\hline Milk & 5,532 & 3,421 & 924 \\
Fat & 5,532 & 3,421 & 924 \\
Protein & 5,532 & 3,421 & 924 \\
Fertility & 4,827 & 3,377 & 941 \\
Birth index & 4,904 & 3,410 & 987 \\
Calving index & 5,317 & 3,359 & 971 \\
Udder health $_{\text {Other diseases }}^{1}$ & 5,531 & 3,421 & 979 \\
Body conformation & 4,957 & 3,409 & 767 \\
Feet and legs & 3,022 & 2,269 & 900 \\
Udder conformation & 3,780 & 2,820 & 899 \\
Milking ability & 3,797 & 2,820 & 901 \\
Temperament & 3,711 & 2,818 & 897 \\
Longevity $_{\text {Yield }}^{2}$ & 3,709 & 2,819 & 896 \\
Average & 5,440 & 3,416 & 635 \\
I Other & 5,532 & 3,421 & 924 \\
& 4,742 & 3,175 & 898 \\
\hline
\end{tabular}

${ }^{1}$ Other diseases: diseases other than udder (includes mainly reproductive and metabolic diseases).

${ }^{2}$ Yield index: $\mathrm{EBV}_{\text {Yield }}=4 \times \mathrm{EBV}_{\text {Protein }}+\mathrm{EBV}_{\text {Fat }}-\mathrm{EBV}_{\text {Milk }}$.

(Table 1) of RDC bulls born from 1980 to 2006. Deregression was carried out using Mix99 package (Lidauer and Strandén, 1999; Strandén and Mäntysaari, 2010). Most of the evaluated bulls were genotyped using the Illumina Bovine SNP50 BeadChip (Illumina, San Diego, CA). After editing SNP data with criteria of minor allele frequency of 0.01 and locus average GenCall score of $0.60,45,168$ SNP markers were used in the genomic analyses. In total, 6,631 bulls had DRP for at least one trait, and of these, 4,408 bulls also had SNP genotypes. The pedigree for the bulls was built by tracing 7 generations back, which led to a pedigree file including 23,358 animals.

The data were divided into a reference data set (training data set) and a test data set. Bulls born before October 1, 2001, were considered reference data, such that approximately the $20 \%$ youngest genotyped bulls were in the test data set for most of traits. It was required that DRP in the reference data set had reliability larger than $10 \%$, and DRP in the test data set had reliability larger than 20\%. Furthermore, only genotyped bulls were kept in the test data set. Thus, the test data set for a given trait was the same for validation of genomic predictions using the different approaches. The numbers of bulls in the reference and test data sets for each trait are shown in Table 1. The total numbers of bulls in reference data sets $\left(\mathbf{R E F}_{\mathbf{t}}\right)$ ranged from 3,022 to 5,532 with an average of 4,742. The numbers of genotyped bulls in reference data sets $\left(\mathbf{R E F}_{\mathbf{s}}\right)$ ranged from 2,269 to 3,421 with an average of 3,175 . The numbers of bulls in test data sets were between 635 and 987 with an average of 898 . The DRP of $\mathrm{REF}_{\mathrm{t}}$ were used to estimate GEBV using a one-step 
blending approach, and the DRP of $\mathrm{REF}_{\mathrm{s}}$ were used to estimate DGV using a linear GBLUP model.

\section{Statistical Analysis}

The DGV were predicted using a linear GBLUP model. Two approaches were used to predict GEBV; one was a selection index blending approach and the other was a one-step blending approach.

Linear GBLUP. The following linear GBLUP model (VanRaden, 2008; Hayes et al., 2009b) was used to predict DGV:

$$
\mathbf{y}=1 \mu+\mathbf{Z g}+\mathbf{e}
$$

where $\mathbf{y}$ was the vector of DRP of genotyped reference bulls, $\mu$ was the overall mean, $\mathbf{g}$ was the vector of additive genetic effects, e was the vector of random residuals, $\mathbf{1}$ was the vector with its elements of 1 , and $\mathbf{Z}$ was the design matrix associating $\mathbf{g}$ with response variables.

Genetic and environmental effects were assumed to follow normal distributions, $\mathbf{g} \sim N\left(\mathbf{0}, \mathbf{G} \sigma_{g}^{2}\right)$ and $e \sim N\left(\mathbf{0}, \mathbf{D} \sigma_{e}^{2}\right)$, where $\sigma_{g}^{2}$ was additive genetic variance, $\mathbf{G}$ was the realized genomic relationship matrix, $\sigma_{e}^{2}$ was residual variance, and $\mathbf{D}$ was a diagonal matrix with element $d_{i i}=1 / w$, where $w$ was a weighting factor for the $i$ th DRP. The weighting factor $w=r_{D R P}^{2} /\left(1-r_{D R P}^{2}\right)$ (VanRaden, 2008; Garrick et al., 2009) was applied to account for heterogeneous residual variances due to different reliabilities of $\operatorname{DRP}\left(r_{D R P}^{2}\right)$.

The genomic relationship matrix was defined as $\mathbf{G}=$ $\mathbf{M M}^{\prime} / \Sigma 2 p_{i} q_{i}$, where $q_{i}$ and $p_{i}$ were the frequencies of allele $1\left(\mathrm{~A}_{1}\right)$ and allele $2\left(\mathrm{~A}_{2}\right)$ at locus $i$, respectively; and $\mathbf{M}$ was $\mathrm{n} \times \mathrm{m}$ matrix $(\mathrm{n}=$ number of animals, $\mathrm{m}$ $=$ number of marker loci) that specified SNP genotype coefficients at each locus. The coefficients of $i$ th column in the $\mathbf{M}$ matrix were $0-2 p_{i}$ for genotype $\mathrm{A}_{1} \mathrm{~A}_{1}, 1-2 p_{i}$ for $\mathrm{A}_{1} \mathrm{~A}_{2}$, and $2-2 p_{i}$ for $\mathrm{A}_{2} \mathrm{~A}_{2}$. The $\mathbf{G}$ matrix was further normalized so that the average of diagonal coefficients was equal to 1 (Forni et al., 2011). The DGV for an individual was defined as $\mathrm{DGV}=\hat{\mathrm{g}}$.

Selection Index Blending. According to VanRaden et al. (2009), the selection index used to estimate GEBV was

$$
\mathrm{GEBV}_{(\mathrm{I})}=\mathrm{b}_{1} \mathrm{DGV}+\mathrm{b}_{2} \mathrm{PI}_{\mathrm{s}}+\mathrm{b}_{3} \mathrm{PI}_{\mathrm{t}}
$$

where $\mathrm{PI}_{t}$ and $\mathrm{PI}_{\mathrm{s}}$ were the traditional EBV or pedigree index estimated from the full data set or from the data used to predict DGV, respectively. In the present study, $\mathrm{PI}_{\mathrm{t}}$ was estimated from the DRP in data set $\mathrm{REF}_{t}$, and
$\mathrm{PI}_{\mathrm{S}}$ was estimated from the DRP in data set $\mathrm{REF}_{\mathrm{s}}$. This meant that $\mathrm{PI} \approx 0.5 \mathrm{EBV}$ of sire $+0.25 \mathrm{EBV}$ of maternal grandsire $+0.125 \mathrm{EBV}$ of maternal great-grandsire for most bulls in the test data set.

The equation system for the selection index was

$$
\begin{aligned}
& \mathrm{b}_{1} \mathrm{~V}_{11}+\mathrm{b}_{2} \mathrm{~V}_{12}+\mathrm{b}_{3} \mathrm{~V}_{13}=\mathrm{V}_{11}, \\
& \mathrm{~b}_{1} \mathrm{~V}_{12}+\mathrm{b}_{2} \mathrm{~V}_{22}+\mathrm{b}_{3} \mathrm{~V}_{23}=\mathrm{V}_{22}, \\
& \mathrm{~b}_{1} \mathrm{~V}_{13}+\mathrm{b}_{2} \mathrm{~V}_{23}+\mathrm{b}_{3} \mathrm{~V}_{33}=\mathrm{V}_{33},
\end{aligned}
$$

where $V_{11}, V_{22}$, and $V_{33}$ were the reliabilities of DGV, $\mathrm{PI}_{\mathrm{t}}$, and $\mathrm{PI}_{\mathrm{s}}$, respectively. We defined $\mathrm{V}_{12}=\mathrm{V}_{22}, \mathrm{~V}_{23}=$ $\mathrm{V}_{22}$, and $\mathrm{V}_{13}=\mathrm{V}_{22}+\left(\mathrm{V}_{11}-\mathrm{V}_{22}\right)\left(\mathrm{V}_{33}-\mathrm{V}_{22}\right) /(1-$ $\mathrm{V}_{22}$ ). To ensure that matrix $\mathbf{V}$ was positive definite, $\mathrm{V}_{11}$ and $V_{33}$ were constrained to be greater than $V_{22}$. In the present study, $\mathrm{V}_{11}, \mathrm{~V}_{22}$, and $\mathrm{V}_{33}$ were obtained by inverting the coefficient matrix of the corresponding model. The expected reliability of the GEBV was $r_{G E B V(I)}^{2}=\mathrm{b}_{1} \mathrm{~V}_{11}+\mathrm{b}_{2} \mathrm{~V}_{22}+\mathrm{b}_{3} \mathrm{~V}_{33}$.

A set of scaling factors ( $S$ ) was used to scale $V_{11}$ (due to possible overestimation of reliabilities of DGV) and DGV (due to possible inflation of DGV) as $\mathrm{V}^{*}{ }_{11}=\mathrm{SV}_{11}$ and $\mathrm{DGV}^{*}=\sqrt{S} D G V$. The detailed results for each trait are presented based on the index with a scaling factor appropriate for most of the traits.

One-Step Blending. Following Legarra et al. (2009), Aguilar et al. (2010), and Christensen and Lund (2010), the one-step blending model had the same form as model [1] but used more information sources. Thus,

$$
\mathbf{y}=1 \mu+\mathbf{Z a}+\mathbf{e},
$$

where $\mathbf{y}$ was the vector of DRP from all reference bulls, including both genotyped and nongenotyped bulls, and a was the vector of additive genetic effects. Because this model used information from all available bulls, the estimated additive genetic value was defined as GEBV. Here, GEBV obtained from this model was denoted as $\operatorname{GEBV}_{(\mathrm{o})}$; that is, $\mathrm{GEBV}_{(\mathrm{o})}=\hat{\mathrm{a}}$.

Genetic values were assumed to follow a normal distribution, $\mathbf{a} \sim N\left(\mathbf{0}, \mathbf{G}_{\mathbf{p}} \sigma_{a}^{2}\right)$, where $\sigma_{a}^{2}$ was additive genetic variance, and $\mathbf{G}_{\mathrm{p}}$ was the genetic relationship matrix constructed by combining information of SNP markers and pedigree. Then,

$$
\begin{gathered}
\mathbf{G}_{\mathbf{P}}=\left[\begin{array}{lc}
\mathbf{G}_{\alpha} & \mathbf{G}_{\alpha} \mathbf{A}_{11}^{-1} \mathbf{A}_{12} \\
\mathbf{A}_{21} \mathbf{A}_{11}^{-1} \mathbf{G}_{\alpha} & \mathbf{A}_{21} \mathbf{A}_{11}^{-1} \mathbf{G}_{\alpha} \mathbf{A}_{11}^{-1} \mathbf{A}_{12}+\mathbf{A}_{22}-\mathbf{A}_{21} \mathbf{A}_{11}^{-1} \mathbf{A}_{12}
\end{array}\right] \\
\text { and } \mathbf{G}_{\alpha}=\alpha \mathbf{G}+(1-\alpha) \mathbf{A}_{11},
\end{gathered}
$$


where $\mathbf{A}_{11}$ was a sub-matrix of pedigree-based relationship matrix (A) for genotyped animals, $\mathbf{A}_{22}$ was a sub-matrix of $\mathbf{A}$ for nongenotyped animals, $\mathbf{A}_{12}$ (or $\mathbf{A}_{21}$ ) was a sub-matrix of $\mathbf{A}$ describing the relationship between genotyped and nongenotyped animals, $\mathbf{G}$ was genomic relationship matrix as defined above, and $\alpha$ was the relative weight on marker-based relationship matrix and $(1-\alpha)$ on pedigree-based relationship coefficients for genotyped animals. The inverse of $\mathbf{G}_{\mathbf{p}}$ was

$$
\mathbf{G}_{\mathbf{p}}^{-1}=\left[\begin{array}{cc}
\mathbf{G}_{\alpha}^{-1}-\mathbf{A}_{11}^{-1} & \mathbf{0} \\
\mathbf{0} & \mathbf{0}
\end{array}\right]+\mathbf{A}^{-1} .
$$

In the present study, the influence of $\alpha$ on GEBV was investigated by varying $\alpha$ from 0.70 to 0.90 . Detailed results for each trait are presented based on the scenario with a relative weight appropriate for most of the traits.

In both the GBLUP and the one-step blending model, variance components were estimated using average information restricted maximum likelihood algorithm (Gilmour et al., 1995; Johnson and Thompson, 1995). The analyses were carried out using the DMU package (Madsen et al., 2010).

\section{Validation of Genomic Prediction}

Reliability of genomic predictions (DGV and GEBV) was assessed as (1) expected reliability obtained by inverting the coefficient matrix of the mixed model equations (Henderson, 1975; Meyer, 1989); that is, modelbased reliability $r_{e}^{2} \approx 1-\frac{P E V}{\sigma_{a}^{2}}$, where $P E V$ was predic- tion error variance; and (2) squared correlation between genomic prediction and DRP for bulls in the test data set, corrected with respect to reliability of average DRP; that is, $r_{v}^{2} \approx \frac{\operatorname{Cov}^{2}(I, D R P)}{\sigma_{I}^{2} \sigma_{D R P}^{2} r_{D R P}^{2}} \approx \frac{\operatorname{Cov}^{2}(I, a)}{\sigma_{I}^{2} \sigma_{a}^{2}}$, where $I$ was genomic prediction, and $a$ was true breeding value, termed as validated reliability in the context. Unbiasedness of genomic predictions was measured as the regression of DRP on the genomic prediction. A necessary condition for unbiased prediction was that the regression coefficient did not deviate significantly from 1 . To make comparison with traditional PI, the same criteria were also applied in PI.

\section{RESULTS}

Heritabilities used in the deregression and average reliabilities of DRP in different data sets are shown in Table 2. Reliabilities of DRP were not completely consistent with heritabilities of the traits, because the sizes of data and the numbers of daughters having records differed among the traits. Reliabilities of DRP for bulls in $\mathrm{REF}_{\mathrm{s}}$ and $\mathrm{REF}_{\mathrm{t}}$ were much higher than those in test data sets for most traits because bulls in the test data sets were younger and had fewer daughters with records or fewer daughters with later lactation records. Reliabilities of DRP for bulls in $\mathrm{REF}_{\mathrm{t}}$ were slightly lower than those in $\mathrm{REF}_{\mathrm{s}}$, indicating that the nongenotyped bulls generally had relatively fewer daughters with records. Averaged over the 15 traits, reliability of DRP was $79.8 \%$ in $\mathrm{REF}_{\mathrm{s}}, 78.5 \%$ in $\mathrm{REF}_{\mathrm{t}}$, and $74.0 \%$ in the test data set.

A set of relative weights $(\alpha=0.70,0.75,0.80,0.85$, and 0.90 ) on marker-based relationship was used to

Table 2. Heritability $\left(\mathrm{h}^{2}\right)$ of the traits, reliability of $\mathrm{DRP}\left(r_{D R P}^{2}\right)$ in total reference data set $\left(\mathrm{REF}_{\mathrm{t}}\right)$, genotyped reference data set $\left(\mathrm{REF}_{\mathrm{s}}\right)$, and test data set

\begin{tabular}{lcccc}
\hline & & $\left(r_{D R P}^{2}\right)$ & $\left(r_{D R P}^{2}\right)$ & $\left(r_{D R P}^{2}\right)$ \\
Trait & $\mathrm{h}^{2}$ & $(\%)$ in $\mathrm{REF}_{\mathrm{t}}$ & $(\%)$ in $\mathrm{REF}_{\mathrm{s}}$ & $(\%)$ in Test \\
\hline Milk & 0.39 & 93.6 & 94.7 & 91.7 \\
Fat & 0.39 & 93.6 & 94.7 & 91.7 \\
Protein & 0.39 & 93.6 & 94.7 & 91.7 \\
Fertility & 0.04 & 76.2 & 78.6 & 67.1 \\
Birth index & 0.06 & 75.7 & 77.0 & 73.7 \\
Calving index & 0.03 & 65.6 & 66.3 & 59.3 \\
Udder health & 0.04 & 88.8 & 90.5 & 79.7 \\
Other diseases & 0.02 & 60.9 & 62.4 & 46.1 \\
Body conformation & 0.30 & 80.0 & 81.2 & 79.1 \\
Feet and legs & 0.10 & 56.1 & 57.4 & 75.4 \\
Udder conformation & 0.25 & 75.0 & 75.9 & 80.7 \\
Milking ability & 0.26 & 79.4 & 73.1 & 70.2 \\
Temperament & 0.13 & 70.8 & 74.9 & 57.4 \\
Longevity & 0.10 & 74.3 & 94.7 & 91.7 \\
Yield & 0.39 & 93.6 & 79.8 & 74.0 \\
Average & 0.19 & 78.5 & & \\
\hline
\end{tabular}


construct the relationship matrix $\mathbf{G}_{\mathrm{p}}$ when using the one-step blending, and a set of scaling factors $(0.80$, $0.85,0.90,0.95$, and 1.00) was used to scale DGV and its reliability when using the selection index blending. As shown in Table 3, averaged over the 15 traits, the Akaike information criterion (Akaike, 1974) decreased with decreasing weight, and reached a minimum at a weight of 0.70 when using one-step blending. In other words, the goodness of fit increased with decreasing effect of genomic-based relationship within the range of weights, and the best fitting was at a weight of 0.70 . The expected reliabilities of GEBV were reduced with decreasing weighting factor in one-step blending and with decreasing scaling factor in selection index blending. The highest validated reliabilities were obtained with weight of 0.80 in one-step blending. However, within the range of these weights, the differences between the validated reliabilities were very small. In selection index blending, scaling had no notable effect on validated reliability. On the other hand, regression coefficients of DRP on GEBV increased considerably with decreasing weight in one-step blending and the scales in selection index blending.

Table 4 presents the expected reliability for $\mathrm{PI}_{\mathrm{t}}, \mathrm{PI}_{\mathrm{s}}$, and DGV, for GEBV from one-step blending with weight on genomic-based relationship being 0.80 , and for GEBV from selection index blending with scale of 0.90 , for each trait. The expected reliabilities for $\mathrm{PI}_{t}$ were slightly higher than those for $\mathrm{PI}_{\mathrm{s}}$, implying a small contribution of nongenotyped bulls to PI of bulls in test data sets. The expected reliabilities for DGV and GEBV were much higher than those for PI, indicating that SNP markers provided more information than pedigree alone. The DGV were predicted from marker information and DRP of bulls in a relatively small data set $\left(\mathrm{REF}_{\mathrm{s}}\right)$, whereas GEBV made use of combined information from markers, pedigree, and DRP of bulls in a large data set $\left(\mathrm{REF}_{\mathrm{t}}\right)$. However, the expected reliabilities of GEBV were lower than reliabilities of DGV. This was because a value of 0.9 was used to scale the expected reliabilities of DGV in the selection index, and a value of 0.8 was used as weight on genomic relationship matrix in the one-step blending model. This also suggested that the expected reliability of DGV could be overestimated.

As shown in Table 5, the validated reliabilities of PI, DGV, and GEBV were much lower than the expected reliability for all traits, except for other diseases. On average, validated reliabilities were lower than the corresponding expected reliabilities by 11 to 13 percentage points for PI and GEBV and by 18 percentage points for DGV. Validated reliabilities ranged from 10.2 to $32.8 \%$ (average $19.9 \%$ ) for $\mathrm{PI}_{\mathrm{t}}, 10.0$ to $30.1 \%$ (average $18.3 \%$ ) for $\mathrm{PI}_{\mathrm{s}}, 16.0$ to $45.4 \%$ (average $30.9 \%$ ) for DGV, 16.1 to $46.7 \%$ (average $31.8 \%$ ) for GEBV from the selection index blending, and from 15.9 to $47.8 \%$ (average $32.2 \%$ ) for GEBV from the one-step blending. The highest validated reliability of genomic prediction was found for fat, possibly due to a known gene (DGAT) with a large effect on fat percentage (Grisart et al., 2004). Averaged over the 15 traits, the validated reliabilities of DGV were 11.0 percentage points higher than reliability of $\mathrm{PI}_{t}$. The further gain of genomic prediction by combining information of traditional PI was 0.9 percentage points using the selection index blending, and 1.3 percentage points using the one-step blending.

Regression coefficients of DRP on genetic predictions for bulls in the test data set are shown in Table 6 . The regression coefficients of DRP on PI ranged from 0.763 to 1.087 with an average of 0.896 for $\mathrm{PI}_{t}$, and from 0.758 to 1.008 with an average of 0.865 for $\mathrm{PI}_{\mathrm{s}}$. The range was between 0.752 and 1.125 with an average of 0.890 for DGV. These results indicated that the variation of PI and DGV was overestimated for most traits (regression coefficients were much lower than 1). By using a weight of 0.8 on the genomic relationship matrix in the one-step blending model and scaling DGV by $\sqrt{0.9}$ and its expected reliability by 0.90 in the selection index, the bias in variation of genetic prediction

Table 3. Akaike information criterion (AIC) for the one-step blending approach, expected reliability $\left(r_{e}^{2}\right)$ and validated reliability $\left(r_{v}^{2}\right)$ of genomic enhanced breeding values (GEBV), and regression coefficient (b) of deregressed proofs (DRP) on GEBV using the one-step blending approach $[\mathrm{GEBV}(\mathrm{o})]$ with different weights on genomic relationships, and using the selection index blending approach [GEBV(I)] with different scaling factors averaged over the 15 traits

\begin{tabular}{|c|c|c|c|c|c|c|c|c|}
\hline \multicolumn{5}{|c|}{ One-step } & \multicolumn{4}{|c|}{ Selection index } \\
\hline Weight & $\mathrm{AIC}$ & $r_{e}^{2} \operatorname{GEBV}(\mathrm{o})(\%)$ & $r_{v}^{2} \operatorname{GEBV(o)}(\%)$ & $\mathrm{b}_{\mathrm{GEBV}(\mathrm{o})}$ & Scale & $r_{e}^{2} \operatorname{GEBV}(\mathrm{I})(\%)$ & $r_{v}^{2} \operatorname{GEBV(I)}(\%)$ & $\mathrm{b}_{\mathrm{GEBV}(\mathrm{I})}$ \\
\hline 0.85 & 43,991 & 45.77 & 32.15 & 0.927 & 0.95 & 47.09 & 31.83 & 0.921 \\
\hline 0.80 & 43,982 & 44.32 & 32.19 & 0.941 & 0.90 & 44.71 & 31.83 & 0.947 \\
\hline 0.75 & 43,978 & 42.91 & 32.16 & 0.953 & 0.85 & 42.33 & 31.82 & 0.973 \\
\hline 0.70 & 43,977 & 41.56 & 32.08 & 0.967 & 0.80 & 40.03 & 31.81 & 1.001 \\
\hline
\end{tabular}


Table 4. Expected reliability of pedigree index from total reference data set $\left(r_{e}^{2}\right.$ PIt $)$, pedigree index from genotyped reference data set $\left(r_{e P I s}^{2}\right)$, direct genomic breeding value from the genomic BLUP model $\left(r_{e D G V}^{2}\right)$, genomic enhanced breeding value from the selection index blending approach $\left(r_{e}^{2} \operatorname{GEBV(I)}\right)$ and from the one-step blending approach $\left(r_{e}^{2} G E B V(o)\right)$

\begin{tabular}{|c|c|c|c|c|c|}
\hline Trait & $r_{e P I t}^{2}(\%)$ & $r_{e P I s}^{2}(\%)$ & $r_{e D G V}^{2}(\%)$ & $r_{e}^{2} \operatorname{GEBV}(I)(\%)^{1}$ & $r_{e}^{2} \operatorname{GEBV(o)}(\%)^{2}$ \\
\hline Milk & 31.6 & 30.2 & 53.5 & 49.1 & 47.9 \\
\hline Fat & 32.1 & 30.8 & 53.5 & 48.9 & 48.6 \\
\hline Protein & 32.2 & 30.9 & 54.0 & 49.5 & 48.8 \\
\hline Fertility & 31.6 & 30.4 & 49.5 & 45.4 & 44.7 \\
\hline Birth index & 28.6 & 27.4 & 44.1 & 40.6 & 39.8 \\
\hline Calving index & 29.9 & 28.5 & 42.2 & 39.1 & 39.2 \\
\hline Udder health & 31.5 & 29.9 & 50.2 & 46.3 & 46.1 \\
\hline Other diseases & 31.8 & 30.2 & 47.1 & 43.5 & 44.1 \\
\hline Body conformation & 31.4 & 29.6 & 46.1 & 42.8 & 42.8 \\
\hline Feet and legs & 29.7 & 28.6 & 42.0 & 38.7 & 38.4 \\
\hline Udder conformation & 32.2 & 30.8 & 49.0 & 45.1 & 45.0 \\
\hline Milking ability & 30.9 & 29.9 & 49.5 & 45.3 & 43.9 \\
\hline Temperament & 29.7 & 28.6 & 44.0 & 40.5 & 40.4 \\
\hline Longevity & 32.2 & 30.8 & 50.7 & 46.6 & 46.0 \\
\hline Yield & 32.3 & 31.0 & 53.9 & 49.4 & 49.0 \\
\hline Average & 31.2 & 29.8 & 48.6 & 44.7 & 44.3 \\
\hline
\end{tabular}

was reduced greatly. Thus, the regressions ranged from 0.821 to 1.139 with an average of 0.946 for GEBV from the selection index blending and from 0.805 to 1.105 with an average of 0.941 for GEBV from the one-step blending.

\section{DISCUSSION}

The present study assessed the accuracy of genomic prediction in RDC. According to the validation analysis, reliability of DGV was higher by 11 percentage points than that of traditional PI, averaged over the 15 traits. Although only one-third of bulls in the present data were nongenotyped, combining information of traditional PI led to a further gain of 0.9 percentage points for genomic prediction using the selection index blending, and 1.3 percentage points using the one-step blending.

The 2 blending approaches used the same information sources but different algorithms. We found that the reliability of GEBV from the one-step blending was slightly higher than that from the selection index blending. The selection index blending involves 2 steps. First, $\mathrm{PI}_{\mathrm{t}}, \mathrm{PI}_{\mathrm{s}}$, and $\mathrm{DGV}$, as well as their reliabilities, are estimated from different data sets and models. Second, these estimates are used to calculate GEBV. Thus, any uncertainty from the first step is not taken into account in the second step (Christensen and Lund, 2010). In the one-step blending, using a combination of genomic relationship matrix and pedigree-based relationship matrix, all information is used to predict
GEBV simultaneously, avoiding several assumptions and parameters required in multiple-step methods (Aguilar et al., 2010; Forni et al., 2011). This is likely why the one-step blending produced more accurate GEBV than the selection index blending. In addition, the one-step blending is easier to implement in practical genetic evaluations and, when applied on data of bull DRP, the computational demand is similar to the demand for predicting DGV using a GBLUP model. On the other hand, selection index blending has the flexibility to increase reliability of GEBV by increasing the accuracy of DGV using more sophisticated models (e.g., Bayesian variable selection models).

In the present study, a set of weights was used to construct $\mathbf{G}_{\mathbf{p}}$ in the one-step blending model and a set of scaling factors was used in the selection index. As shown in Table 6 , the average regression coefficient of DRP on DGV over the 15 traits was 0.890 , indicating an inflation of DGV. Inflation of genetic evaluations using genomic information would cause top young bulls to have an unfair advantage over older progenytested bulls (Aguilar et al., 2010). Using a weight of 0.80 on genomic relationship in the one-step blending model and a scale of 0.9 for DGV and its reliability in the selection index seemed appropriate in the present analyses, according to the validation analysis. First, the regression of DRP on GEBV increased to be 0.941 (one-step blending) and 0.946 (selection index). Second, these factors resulted in the highest reliability of GEBV averaged over the 15 traits. This showed the impor- 
Table 5. Validated reliability of pedigree index from total reference data set $\left(r_{v P I t}^{2}\right)$, pedigree index from genotyped reference data set $\left(r_{v P I s}^{2}\right)$, direct genomic breeding value from the genomic BLUP model $\left(r_{v D G V}^{2}\right)$, genomic enhanced breeding value from the selection index blending approach $\left(r_{v}^{2} G E B V(I)\right)$ and from the one-step blending approach $\left(r_{v}^{2} \operatorname{GEBV(o)}\right)$

\begin{tabular}{lccccc} 
Trait & $r_{v P I t}^{2}(\%)$ & $r_{v P I s}^{2}(\%)$ & $r_{v D G V}^{2}(\%)$ & $r_{v}^{2}$ & GEBV $(I)$ \\
\hline Milk & 19.4 & 18.1 & 35.8 & 36.2 & $r_{v}^{2} G_{G E B V(o)}(\%)^{2}$ \\
Fat & 25.1 & 22.8 & 45.4 & 46.7 & 36.7 \\
Protein & 19.9 & 16.7 & 34.6 & 36.3 & 47.8 \\
Fertility & 16.6 & 15.7 & 29.7 & 30.9 & 37.2 \\
Birth index & 10.2 & 10.0 & 19.5 & 19.7 & 19.8 \\
Calving index & 11.1 & 11.0 & 16.0 & 16.1 & 15.9 \\
Udder health & 17.1 & 14.4 & 24.4 & 26.3 & 26.2 \\
Other diseases & 32.8 & 30.1 & 30.1 & 32.0 & 32.9 \\
Body conformation & 30.6 & 28.4 & 45.0 & 45.8 & 45.5 \\
Feet and legs & 14.8 & 16.6 & 29.6 & 27.8 & 26.5 \\
Udder conformation & 23.5 & 23.0 & 32.1 & 31.7 & 32.0 \\
Milking ability & 13.9 & 12.7 & 29.7 & 30.7 & 30.1 \\
Temperament & 18.9 & 18.6 & 30.0 & 29.8 & 29.6 \\
Longevity & 24.1 & 19.8 & 25.9 & 29.4 & 32.5 \\
Yield & 20.6 & 17.2 & 36.1 & 37.9 & 38.8 \\
Average & 19.9 & 18.3 & 30.9 & 31.8 & 32.2 \\
\hline
\end{tabular}

${ }^{1} \mathrm{GEBV}(\mathrm{I})$ was obtained from the selection index blending where $r_{D G V}^{2}$ was scaled down by 0.90 and DGV by $\sqrt{0.9}$.

${ }^{2} \mathrm{GEBV}(\mathrm{o})$ was obtained from the one-step blending with $\alpha=0.80$.

tance of choosing appropriate weight or scaling factors in blending procedures. Aguilar et al. (2010) found the highest regression coefficient $(0.92)$ at a weight $(\lambda)$ of 0.5 in their single-step model for final score in US Holstein, but with a 2 percentage point loss in reliability of GEBV. Alternatively, Liu et al. (2011) reported that bias of genomic prediction could be reduced by including a residual polygenic effect in a SNP-BLUP model and suggested that the optimal proportions of residual polygenic variance to total additive genetic variance would be between 5 and $10 \%$ for most traits. Christensen and Lund (2010) proposed to determine weight in a single-step model according to likelihood of REML analysis and found that a weight close to 1 was appropriate in their stimulation data. However, likelihood from REML analysis measures the goodness of fit but

Table 6. Regression coefficients of deregressed proofs (DRP) on pedigree index from total reference data set $\left(b_{P I t}\right)$, pedigree index from genotyped reference data set $\left(b_{P I s}\right)$, direct genomic breeding value from the genomic BLUP model $\left(b_{D G V}\right)$, genomic enhanced breeding value from the selection index blending approach $\left(b_{G E B V(I)}\right)$, and from the one-step blending approach $\left(b_{G E B V(o)}\right)$

\begin{tabular}{|c|c|c|c|c|c|}
\hline Trait & $b_{P I t}$ & $b_{P I s}$ & $b_{D G V}$ & $b_{G E B V(I)}^{1}$ & $b_{G E B V(o)^{2}}$ \\
\hline Milk & 0.840 & 0.810 & 0.863 & 0.912 & 0.913 \\
\hline Fat & 0.955 & 0.919 & 0.969 & 1.025 & 1.024 \\
\hline Protein & 0.869 & 0.781 & 0.849 & 0.911 & 0.918 \\
\hline Fertility & 0.964 & 0.951 & 0.934 & 1.006 & 1.024 \\
\hline Birth index & 0.890 & 0.894 & 0.984 & 1.039 & 1.044 \\
\hline Calving index & 0.887 & 0.887 & 0.941 & 0.993 & 0.954 \\
\hline Udder health & 0.921 & 0.885 & 0.851 & 0.908 & 0.886 \\
\hline Other diseases & 0.950 & 0.929 & 0.782 & 0.836 & 0.811 \\
\hline Body conformation & 0.896 & 0.900 & 0.958 & 0.989 & 0.959 \\
\hline Feet and legs & 0.937 & 1.008 & 1.125 & 1.139 & 1.105 \\
\hline Udder conformation & 0.809 & 0.814 & 0.798 & 0.821 & 0.805 \\
\hline Milking ability & 0.763 & 0.758 & 0.832 & 0.877 & 0.886 \\
\hline Temperament & 0.803 & 0.803 & 0.842 & 0.880 & 0.864 \\
\hline Longevity & 1.087 & 0.846 & 0.752 & 0.926 & 0.978 \\
\hline Yield & 0.874 & 0.790 & 0.870 & 0.933 & 0.936 \\
\hline Average & 0.896 & 0.865 & 0.890 & 0.946 & 0.941 \\
\hline
\end{tabular}


not the accuracy and unbiasedness of prediction. For predicting genetic merit of the candidates without their own or offspring records, it is necessary to perform a validation analysis to assess an appropriate weight.

As mentioned above, a weight of 0.80 in the onestep blending and a scale of 0.90 in the selection index blending led to highest validated reliability of GEBV without serious inflation, averaged over the 15 traits. However, the weight and scale were not optimal for every single trait with regard to reliability and unbiasedness of GEBV. For example, smaller values might be better for the traits udder confirmation and other diseases. For these 2 traits, the regression coefficients of DRP on GEBV from selection index blending were 0.811 and 0.836 , and those on GEBV from one-step blending were 0.805 and 0.811 , at current weight and scale. Because the optimal weight and scale for different traits could be different, it could be beneficial to use trait-specific weight and scale in the one-step and the selection index blending approaches. Similarly, Liu et al. (2011) reported that the optimal partitions of the additive genetic variance into the residual polygenic and SNP-based components were trait-dependent in their analysis using a SNP-BLUP model, including SNP effects and residual polygenic effects. In addition, we observed in the current study that PI was also inflated. To get more accurate and unbiased GEBV, more sophisticated weighting and scaling strategies in a blending procedure are required.

The present study showed that the validated reliabilities were much lower than the expected reliabilities with the largest difference for DGV. This suggested that either the expected reliabilities overestimated the true reliabilities or the validated reliabilities underestimated the true reliability or both (VanRaden et al., 2009; Su et al., 2010). The expected reliabilities might be overestimated if the markers cannot explain all additive genetic variance or if the markers overfit the data. On the other hand, reliability might be underestimated in the validation analysis. The validated reliabilities were measured as the squared correlation divided by reliability of DRP for bulls in the test data. The measure of reliability was unbiased only if the validation bulls were a random sample. However, the bulls in this study were selected from elite parents based on PI. This directional selection would reduce the correlation between PI and genomic predicted breeding values and consequently, underestimate the reliabilities of genomic predictions. The underestimation would be most severe for strongly selected traits. Uimari and Mäntysaari (1993) determined that $10 \%$ selection based on PI reduced the expected correlation between PI and daughter-based EBV by half; that is, from 0.62 to 0.31 . VanRaden et al. (2009) proposed to measure reliability of genomic predictions as validated reliability of genomic prediction plus the difference between expected and validated reliabilities of PI. Using this procedure, the mean reliability of the 15 traits was $43.1 \%$ for GEBV from the selection index blending and $43.5 \%$ from the one-step blending, which was very close to the expected reliabilities. However, this measure was only valid if the expected reliability of PI was unbiased. Given the arguments above, it was reasonable to assume that the true reliabilities of genomic predictions in the present study were in the range between the validated and the expected reliabilities.

The one-step blending approach in the present study is not a regular single-step approach (Misztal et al., 2009; Aguilar et al., 2010; Christensen and Lund, 2010). The single-step approach predicts GEBV based on original phenotypic records of the whole population, and therefore avoids uncertainty arising during the steps from original phenotypic records to DRP, and enables the use of information of bull dams. In addition, the regular single-step model can avoid prediction bias due to preselection of young animals on Mendelian sampling variations. In general, the regular single-step approach does not cost much additional time compared with traditional BLUP model with pedigree-based relationship matrix (Tsuruta et al., 2011). However, it might have a high computational demand in the case of large data set applying complex models such as a multi-trait test-day model. Moreover, it is debatable whether it is desirable to include records of bull dams in the data for genomic predictions, because the records might be biased due to preferential treatment (Uimari and Mantysaari, 1993; Aguilar et al., 2010), especially for yield traits. In contrast to the regular single-step model, the proposed one-step blending is easier to implement in routine genomic evaluations. In addition to its low computational demands, the one-step blending is convenient for genomic prediction when a reference population includes genotyped foreign bulls.

\section{CONCLUSIONS}

The results from this study indicate that genomic selection can greatly improve the accuracy of preselection for young bulls, and reliability of genomic prediction can be improved by combining information of traditional EBV or nongenotyped animals. The one-step blending approach is a natural procedure to integrate information of genotyped and nongenotyped animals through a relationship matrix combining marker and pedigree information; it is also easy to implement. Therefore, the one-step blending approach could be a good alternative to predict GEBV in practical genetic evaluation program. 


\section{ACKNOWLEDGMENTS}

We thank the Danish Cattle Federation (Aarhus, Denmark), Faba Co-op (Hollola, Finland), Swedish Dairy Association (Stockholm, Sweden), and Nordic Cattle Genetic Evaluation (Aarhus, Denmark) for providing data. This work was performed in the project "Genomic Selection-From function to efficient utilization in cattle breeding" (grant no. 3405-10-0137), funded under Green Development and Demonstration Programme by the Danish Directorate for Food, Fisheries and Agri Business (Copenhagen, Denmark), the Milk Levy Fund (Aarhus, Denmark), VikingGenetics (Randers, Denmark), Nordic Cattle Genetic Evaluation (Aarhus, Denmark), and Aarhus University (Aarhus, Denmark).

\section{REFERENCES}

Aguilar, I., I. Misztal, D. L. Johnson, A. Legarra, S. Tsuruta, and T. J. Lawlor. 2010. Hot topic: A unified approach to utilize phenotypic, full pedigree, and genomic information for genetic evaluation of Holstein final score. J. Dairy Sci. 93:743-752.

Akaike, H. 1974. A new look at the statistical model identification. IEEE Trans. Automat. Contr. 19:716-723.

Christensen, O. F., and M. S. Lund. 2010. Genomic prediction when some animals are not genotyped. Genet. Sel. Evol. 42:2.

Cole, J. B., P. M. VanRaden, J. R. O'Connell, C. P. Van Tassell, T. S. Sonstegard, R. D. Schnabel, J. F. Taylor, and G. R. Wiggans. 2009. Distribution and location of genetic effects for dairy traits. J. Dairy Sci. 92:2931-2946.

Forni, S., I. Aguilar, and I. Misztal. 2011. Different genomic relationship matrices for single-step analysis using phenotypic, pedigree and genomic information. Genet. Sel. Evol. 43:1.

Garrick, D. J., J. F. Taylor, and R. L. Fernando. 2009. Deregressing estimated breeding values and weighting information for genomic regression analyses. Genet. Sel. Evol. 41:55.

Gilmour, A. R., R. Thompson, and B. R. Cullis. 1995. Average information REML: An efficient algorithm for variance parameter estimation in linear mixed models. Biometrics 51:1440-1450.

Goddard, M. 1985. A method of comparing sires evaluated in different countries. Livest. Prod. Sci. 13:321-331.

Goddard, M. E., and B. J. Hayes. 2009. Mapping genes for complex traits in domestic animals and their use in breeding programmes. Nat. Rev. Genet. 10:381-391.

Grisart, B., F. Farnir, L. Karim, N. Cambisano, J. J. Kim, A. Kvasz, M. Mni, P. Simon, J. M. Frere, W. Coppieters, and M. Georges. 2004. Genetic and functional confirmation of the causality of the DGAT1 K232A quantitative trait nucleotide in affecting milk yield and composition. Proc. Natl. Acad. Sci. USA 101:2398-2403.

Guo, G., M. S. Lund, Y. Zhang, and G. Su. 2010. Comparison between genomic predictions using daughter yield deviation and conventional estimated breeding value as response variables. J. Anim. Breed. Genet. 127:423-432.

Harris, B. L., and D. L. Johnson. 2010. Genomic predictions for New Zealand dairy bulls and integration with national genetic evaluation. J. Dairy Sci. 93:1243-1252.

Hayes, B. J., P. J. Bowman, A. J. Chamberlain, and M. E. Goddard. 2009a. Invited review: Genomic selection in dairy cattle: Progress and challenges. J. Dairy Sci. 92:433-443.

Hayes, B. J., P. M. Visscher, and M. E. Goddard. 2009b. Increased accuracy of artificial selection by using the realized relationship matrix. Genet. Res. (Camb.) 91:47-60.
Henderson, C. R. 1975. Best linear unbiased estimation and prediction under a selection model. Biometrics 31:423-447.

Jairath, L., J. C. M. Dekkers, L. R. Schaeffer, Z. Liu, E. B. Burnside, and B. Kolstad. 1998. Genetic evaluation for herd life in Canada. J. Dairy Sci. 81:550-562.

Johnson, D. L., and R. Thompson. 1995. Restricted maximum-likelihood-estimation of variance-components for univariate animalmodels using sparse-matrix techniques and average information. J. Dairy Sci. 78:449-456.

Legarra, A., I. Aguilar, and I. Misztal. 2009. A relationship matrix including full pedigree and genomic information. J. Dairy Sci. 92:4656-4663.

Lidauer, M., and I. Strandén. 1999. Fast and flexible program for genetic evaluation in dairy cattle. Interbull Bull. 20:19-24.

Liu, Z. T., F. R. Seefried, F. Reinhardt, S. Rensing, G. Thaller, and R. Reents. 2011. Impacts of both reference population size and inclusion of a residual polygenic effect on the accuracy of genomic prediction. Genet. Sel. Evol. 43:19.

Lund, M. S., A. P. W. de Roos, A. G. de Vries, T. Druet, V. Ducrocq, S. Fritz, F. Guillaume, B. Guldbrandtsen, Z. Liu, R. Reents, C. Schrooten, M. Seefried, and G. Su. 2010. Improving genomic prediction by EuroGenomics collaboration. In Proc. 9th World Congr. Genet. Appl. Livest. Prod., Leipzig, Germany, paper 880. Gesellschaft für Tierzuchtwissenschaft e.V., Bonn, Gemany.

Lund, M. S., G. Sahana, D. J. de Koning, G. Su, and Ö. Carlborg. 2009. Comparison of analyses of the QTLMAS XII common dataset. I: Genomic selection. BMC Proc. 3:S1.

Madsen, P., G. Su, R. Labouriau, and O. F. Christensen. 2010. DMUA package for analyzing multivariate mixed models. In Proc. 9th World Congr. Genet. Appl. Livest. Prod., Leipzig, Germany, paper 732. Gesellschaft für Tierzuchtwissenschaft e.V., Bonn, Gemany.

Meuwissen, T. H. E., and M. E. Goddard. 2004. Mapping multiple QTL using linkage disequilibrium and linkage analysis information and multitrait data. Genet. Sel. Evol. 36:261-279.

Meuwissen, T. H. E., B. J. Hayes, and M. E. Goddard. 2001. Prediction of total genetic value using genome-wide dense marker maps. Genetics 157:1819-1829.

Meyer, K. 1989. Approximate accuracy of genetic evaluation under an animal-model. Livest. Prod. Sci. 21:87-100.

Misztal, I., A. Legarra, and I. Aguilar. 2009. Computing procedures for genetic evaluation including phenotypic, full pedigree, and genomic information. J. Dairy Sci. 92:4648-4655.

Schaeffer, L. R. 1985. Model for international evaluation of dairy sires. Livest. Prod. Sci. 12:105-115.

Schaeffer, L. R. 2001. Multiple trait international bull comparisons. Livest. Prod. Sci. 69:145-153.

Strandén, I., and E. Mäntysaari. 2010. A recipe for multiple trait deregression. Interbull Bull. 42:21-24.

$\mathrm{Su}$, G., B. Guldbrandtsen, V. R. Gregersen, and M. S. Lund. 2010. Preliminary investigation on reliability of genomic estimated breeding values in the Danish Holstein population. J. Dairy Sci. 93:1175-1183.

Tsuruta, S., I. Misztal, I. Aguilar, and T. J. Lawlor. 2011. Multipletrait genomic evaluation of linear type traits using genomic and phenotypic data in US Holsteins. J. Dairy Sci. 94:4198-4204.

Uimari, P., and E. A. Mantysaari. 1993. Repeatability and bias of estimated breeding values for dairy bulls and bull dams calculated from animal-model evaluations. Anim. Prod. 57:175-182.

VanRaden, P. M. 2008. Efficient methods to compute genomic predictions. J. Dairy Sci. 91:4414-4423.

VanRaden, P. M., and P. G. Sullivan. 2010. International genomic evaluation methods for dairy cattle. Genet. Sel. Evol. 42:7.

VanRaden, P. M., C. P. Van Tassell, G. R. Wiggans, T. S. Sonstegard, R. D. Schnabel, J. F. Taylor, and F. S. Schenkel. 2009. Invited review: Reliability of genomic predictions for North American Holstein bulls. J. Dairy Sci. 92:16-24.

Villumsen, T. M., L. Janss, and M. S. Lund. 2009. The importance of haplotype length and heritability using genomic selection in dairy cattle. J. Anim. Breed. Genet. 126:3-13. 